\title{
Lipoma intraoral atípico: relato de caso
}

\author{
Atipical intraoral lipoma: case report \\ Lipoma intraoral atipico: reporte de caso \\ Naíza Menezes Medeiros ABRAHIM ${ }^{1}$ \\ Gesom Avohai Dias SOMBRA ${ }^{2}$ \\ Jardel dos Santos SILVA ${ }^{2}$ \\ André Luiz Carvalho BARREIROS ${ }^{3}$ \\ Jeconias CÂMARA \\ Luciana Botinelly Mendonça FUJIMOTO ${ }^{5}$ \\ ${ }^{1}$ Mestre em cirurgia, Faculdade de Medicina, Departamento de Patologia e Medicina Legal, Universidade Federal do Amazonas, $69020-160$ - AM, Brasil \\ ${ }^{2}$ Graduação em odontologia na Universidade Federal do Amazonas, 69025-050 - AM, Brasil \\ ${ }^{3}$ Mestre em Odontologia, CPO, São Leopoldo/SP, Brasil \\ ${ }^{4}$ Mestre em Patologia Oral, Faculdade de Medicina, Departamento de Patologia e Medicina Legal, Universidade Federal do Amazonas, $69020-160$ - AM, Brasil \\ ${ }_{5}^{5}$ Doutora em Biotecnologia, Faculdade de Medicina, Departamento de Patologia e Medicina Legal, Universidade Federal do Amazonas, $69020-160$ - AM, Brasil
}

\section{Resumo}

Os lipomas são tumores de tecido mole que raramente acometem a cavidade oral. A lesão apresenta variáveis clínicas e histopatológicas que não alteram seu prognóstico. Apesar de suas características comuns, a lesão pode estabelecer diagnóstico diferencial com outras lesões de tecido mole. O objetivo desse artigo é relatar a apresentação clínica não usual de um lipoma localizado em mucosa jugal com $3 \mathrm{~cm}$ em sua maior extensão, em paciente do sexo masculino com 56 anos de idade. O plano de tratamento foi a biópsia excisional com excelente prognóstico.

Descritores: Boca; Lipoma; Neoplasias Bucais.

\section{Abstract}

Lipomas are soft tissue tumors that rarely involve the oral cavity. The lesion presents clinical and histopathological variables that do not alter its prognosis. Despite its common characteristics, the lesion may establish differential diagnosis with other soft tissue lesions. The aim this article is to report the unusual clinical presentation of a lipoma located in the buccal mucosa with $3 \mathrm{~cm}$ in its greatest extension, in a 56 year old male patient. The treatment plan was excisional biopsy with an excellent prognosis.

Descriptors: Mouth; Lipoma; Mouth Neoplasms.

\section{Resumen}

Los lipomas son tumores de tejidos blandos que rara vez afectan la cavidad oral. La lesión presenta variables clínicas e histopatológicas que no alteran su pronóstico. A pesar de sus características comunes, la lesión puede establecer un diagnóstico diferencial con otras lesiones de tejidos blandos. El objetivo de este trabajo es informar la presentación clínica inusual de un lipoma ubicado en la mucosa yugal de $3 \mathrm{~cm}$ de largo en un paciente masculino de 56 años. El plan de tratamiento fue la biopsia por escisión con excelente pronóstico.

Descriptores: Boca; Lipoma; Neoplasias de la Boca.

\section{INTRODUÇÃO}

Os lipomas são tumores mesenquimais benignos compostos por células adiposas maduras, que podem ocorrer em qualquer parte do corpo. 15 a $20 \%$ dos casos envolvem a região de cabeça e pescoço, em contrapartida com a região intrabucal, em que a lesão é relativamente rara afetando apenas em 1 a $4 \%$ dos casos. As regiões intraorais mais afetadas são a mucosa jugal, lábios, língua, palato, vestíbulo, assoalho bucal e região retromolar ${ }^{1-4}$.

A etiologia e patogênese dos lipomas ainda não é clara, porém traumas, fatores genéticos, alterações endócrinas ou inflamatórias, entre outros fatores causais são apontadas na literatura ${ }^{5-7}$. Os lipomas apresentam variações histológicas, sendo classificados em: lipoma simples, fibrolipoma, lipoma de células fusiformes, lipoma intramuscular, condrolipoma, lipoma pleomórfico, lipoma mixóide, angiolipoma e sialolipoma ${ }^{5,6}$.

Os tumores são circunscritos, indolores e de crescimento lento, recobertos por mucosa, acometendo principalmente regiões onde há acúmulo de gordura ${ }^{5,6}$. A base pode ser séssil ou pediculada, a coloração amarelada e a consistência variando de suave a firme. Pequenos lipomas intraorais podem ser assintomáticos, todavia grandes tumores podem ocasionar problemas mastigatórios e desconforto, disfagia e dispneia ${ }^{7}$.

O diagnóstico se dá após biópsia excisional e laudo histopatológico. A lesão tende a flutuar ao ser depositada em solução de formol a 10\%, o que reafirma o seu diagnóstico clínico. O tratamento consiste na excisão local da lesão, sendo incomum a sua recorrência ${ }^{7,8}$.

O presente artigo tem como objetivo relatar o caso de um portador de lipoma intraoral em região de mucosa jugal tratado com excisão cirúrgica, salientando características clínicas e histopatológicas desta lesão.

\section{CASO CLÍNICO}

Paciente do sexo masculino, 56 anos, leucoderma e tabagista, queixou-se de aumento de volume em mucosa jugal esquerda, sem sintomatologia dolorosa e com evolução de aproximadamente cinco anos. Clinicamente notou-se a presença de massa exofítica pediculada na região de mucosa jugal, consistência firme, aspecto liso, medindo $3 \mathrm{~cm}$ de diâmetro em sua maior extensão. A coloração da lesão era rosácea, semelhante a mucosa, porém com áreas branco-acinzentadas, não sangrantes (Figuras 1 e 2 ). A hipótese clínica diagnóstica foi de lipoma intraoral. Diante deste quadro, optou-se por realizar a biópsia excisional da lesão com anestesia local. Notou-se que ao colocar-se a peça cirúrgica em solução de formol a $10 \%$, a mesma flutuou, sugerindo a presença de células 
adiposas. O material seguiu para a análise histopatológica, sendo estabelecido o diagnóstico de lipoma. Os cortes histológicos exibiram células com citoplasma volumoso e núcleos deslocados para a periferia (adipócitos).

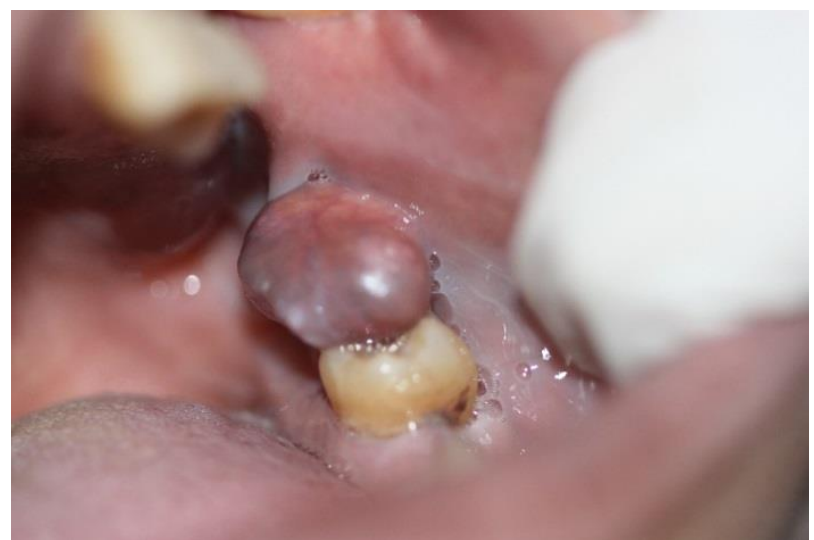

Figura 1: Aspecto clínico da lesão observando-se lesão nodular em mucosa jugal esquerda de superfície lisa, pediculada, coloração rosada com áreas branco-acizentadas, com aproximadamente $3 \mathrm{~cm}$ em sua maior extensão.

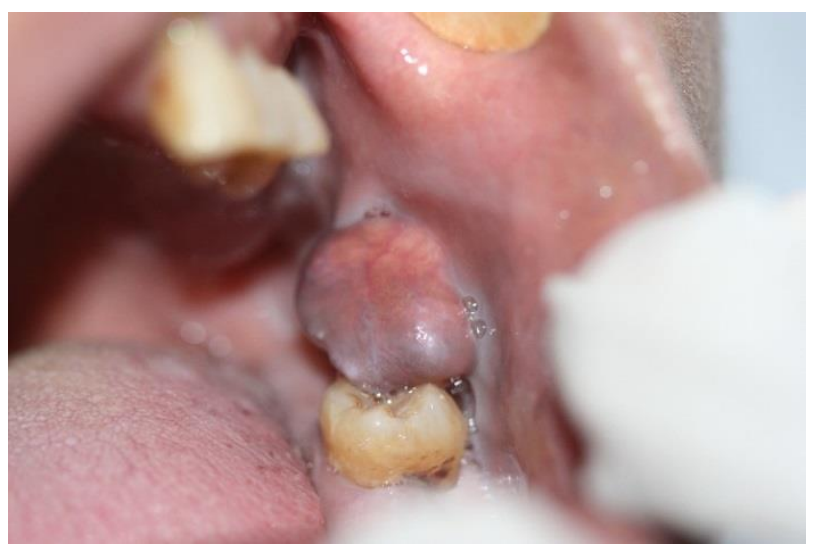

Figura 2: Aspecto clínico da lesão observando-se lesão nodular em mucosa jugal esquerda de superfície lisa, pediculada, coloração rosada com áreas branco-acizentadas, com aproximadamente $3 \mathrm{~cm}$ em sua maior extensão.

A superfície epitelial apresentou hiperqueratose, sendo separada da neoplasia benigna repleta de adipócitos por uma cápsula fibrosa (Figuras 3 a 5). A paciente teve alta após a remoção da sutura, e em oito meses do tratamento a encontrase sem sinais de recidiva.

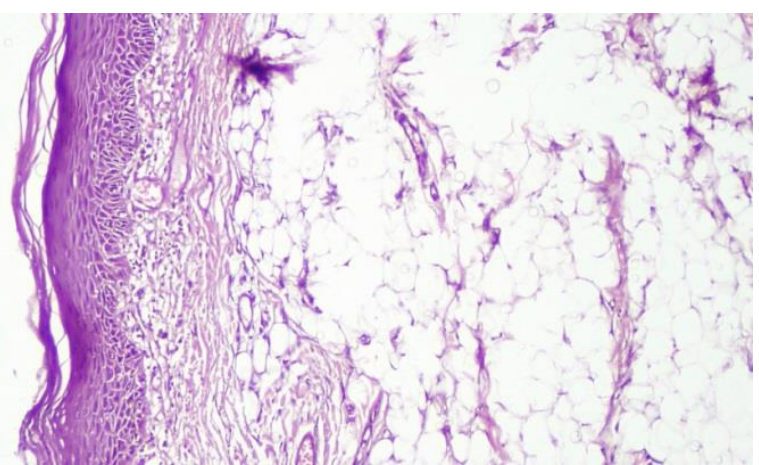

Figura 3: Aspecto microscópico da biópsia excisional exibindo neoplasia benigna constituída de adipócitos separada do epitélio de superfície suprajacente por uma cápsula de tecido conjuntivo, a superfície epitelial exibe hiperqueratose.

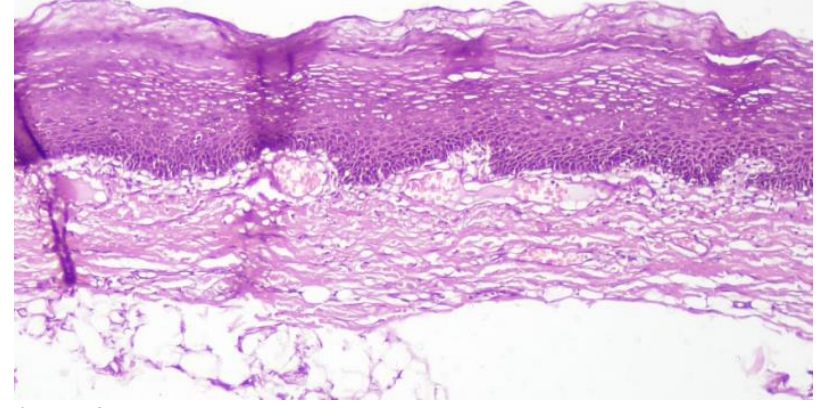

Figura 4: Aspecto microscópico da biópsia excisional exibindo neoplasia benigna constituída de adipócitos separada do epitélio de superfície suprajacente por uma cápsula de tecido conjuntivo, a superfície epitelial exibe hiperqueratose.

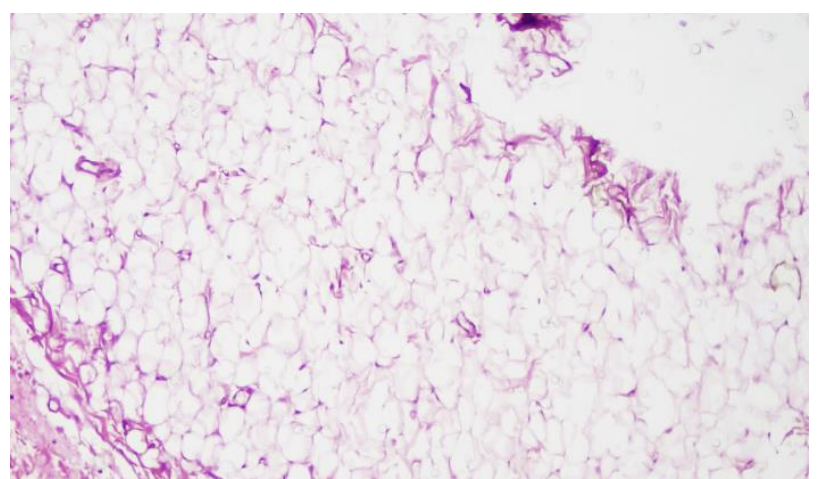

Figura 5: Aspecto microscópico da biópsia excisional exibindo no maior aumento, células apresentando citoplasma volumoso com núcleos deslocados para a periferia (adipócitos).

\section{RESULTADOS E DISCUSSÃO}

Em nossa revisão de literatura foram selecionados estudos restropectivos que analisaram aproximadamente 311 casos de lipomas. A faixa etária variou de 18 a 91 anos, com a mucosa jugal sendo o local mais acometido (Tabela 1).

Tabela 1. Resumo dos 311 casos de lipoma oral relatados na literatura

\begin{tabular}{|c|c|c|c|c|c|c|}
\hline & $\begin{array}{c}\text { Juliasse } \\
\text { et al } \\
(2010)\end{array}$ & $\begin{array}{l}\text { Naruse } \\
\text { et al } \\
(2015)\end{array}$ & $\begin{array}{c}\text { Manor } \\
\text { et al } \\
(2011)\end{array}$ & $\begin{array}{c}\text { Avelar } \\
\text { et al } \\
(2008)\end{array}$ & $\begin{array}{c}\text { Said-Al- } \\
\text { Naief et al } \\
(2001)\end{array}$ & TOTAL \\
\hline $\begin{array}{l}\text { Número de } \\
\text { casos }\end{array}$ & 41 & 24 & 58 & 42 & 146 & 311 \\
\hline $\begin{array}{c}\text { Período de } \\
\text { estudo (anos) }\end{array}$ & 38 & 33 & 20 & 16 & N/D & N/D \\
\hline \multicolumn{7}{|l|}{ Sexo } \\
\hline Masculino & 12 & 11 & 29 & 24 & 77 & 153 \\
\hline Feminino & 29 & 13 & 29 & 18 & 69 & 158 \\
\hline Faixa etária & $18-75$ & $31-90$ & N/D & $51-60$ & N/D & N/D \\
\hline $\begin{array}{l}\text { Tamanho } \\
\text { médio }(\mathrm{cm})\end{array}$ & 2,1 & 2 & 1,68 & 2,53 & N/D & N/D \\
\hline \multicolumn{7}{|l|}{ Localizacão } \\
\hline Mucosa jugal & 22 & 9 & 31 & 22 & 49 & 133 \\
\hline Vestibulo & 6 & o & 5 & 0 & 14 & 25 \\
\hline Gengiva & o & 1 & 0 & o & 0 & 1 \\
\hline Área Retromolar & 3 & 2 & 0 & 4 & 9 & 18 \\
\hline Língua & 4 & 4 & 10 & 0 & 21 & 39 \\
\hline Lábio & 1 & 3 & 6 & 8 & 31 & 49 \\
\hline Assoalho bucal & 3 & 2 & 6 & o & 8 & 19 \\
\hline Palato & 1 & 3 & 0 & o & 8 & 12 \\
\hline Outros & 1 & 0 & 0 & 8 & 6 & 15 \\
\hline \multicolumn{7}{|l|}{$\begin{array}{c}\text { Subtipo } \\
\text { histológico }\end{array}$} \\
\hline Lipoma & 18 & 20 & 28 & 39 & 83 & 188 \\
\hline Fibrolipoma & 14 & 2 & 19 & 2 & 50 & 87 \\
\hline Lipoma mixoide & 0 & o & 0 & o & 8 & 8 \\
\hline Angiolipoma & o & o & 2 & 1 & 3 & 6 \\
\hline Intramuscular & 0 & 1 & 4 & 0 & 0 & 5 \\
\hline $\begin{array}{l}\text { Lipoma de células } \\
\text { fusiformes }\end{array}$ & 4 & 1 & 3 & 0 & 2 & $\begin{array}{c}5 \\
10\end{array}$ \\
\hline $\begin{array}{c}\text { Lipoma de } \\
\text { glândula salivar } \\
\text { menor }\end{array}$ & 4 & 0 & 2 & 0 & 0 & 6 \\
\hline $\begin{array}{l}\text { Duração } \\
\text { (meses) }\end{array}$ & 48 & 12 & 31,2 & N/D & N/D & N/D \\
\hline $\begin{array}{c}\text { Seguimento } \\
\text { (meses) }\end{array}$ & 45 & 3 & 31,5 & N/D & $12-96$ & N/D \\
\hline
\end{tabular}


Os lipomas são neoplasias mesenquimais adiposas que embora muito comuns na região de cabeça e pescoço, são relativamente raras na cavidade oral. A literatura descreve uma prevalência que varia de 1 a $4 \%^{1-4}$. A lesão afeta frequentemente indivíduos acima dos 40 anos de idade, com maior prevalência no sexo masculino, no entanto, alguns estudos mostram não haver diferenças quando comparado ao sexo feminino ${ }^{3,4,8}$. Os estudos retrospectivos realizados por Juliasse et al. ${ }^{1}$ e por Naruse et al ${ }^{2}$ observaram discreta predileção pelo sexo feminino. Embora os lipomas tenham maior incidência em pessoas obesas, sabe-se que a lesão independe do metabolismo lipídico ${ }^{8,9}$.

Os lipomas são mais comuns na mucosa jugal, uma região rica em tecido adiposo, porém qualquer região da cavidade bucal pode ser acometida ${ }^{3,4,7}$. Uma revisão realizada por EgidoMoreno et al. ${ }^{6}$ avaliou 95 lipomas intraorais, onde demonstrou uma leve predisposição desta neoplasia pelo sexo feminino, além de estabelecer a idade média de 52 anos, afetando principalmente pacientes da quarta a sexta década de vida. Quanto a localização anatômica da lesão, a mucosa jugal foi a mais afetada, seguida pela língua, lábios, palato e assoalho bucal. O caso apresentado ocorreu em paciente do sexo masculino, 56 anos, com faixa etária compatível com a literatura.

No ponto de vista clínico, os lipomas intraorais exibem comportamento benigno, que se traduz em crescimento lento, geralmente assintomático. As lesões mostram-se como nódulos submucosos indolores, móveis, com coloração amarelada. A maioria das lesões tem tempo de evolução que variam de 6 meses a $15 \operatorname{anos}^{4,7-9}$. Em nosso caso, paciente relatou a evolução da lesão por um período de 5 anos. A característica da lesão apresentou rosada, ao invés de amarelada, como descrita na maioria dos casos da literatura. Isso normalmente ocorre devido a profundidade da lesão O tamanho médio dos lipomas é de 0,5 a $8 \mathrm{~cm}$, com média de aproximadamente $2 \mathrm{~cm}^{1-4}$. Em nosso caso, a lesão apresentou tamanho acima da média, com $3 \mathrm{~cm}$ de diâmetro na sua maior extensão.

Apesar de serem incomuns em região oral, o diagnóstico clínico dos lipomas é facilitado devido características como coloração amarelada, consistência e localização usual específica ${ }^{6,9}$. Apesar dessas características, a lesão pode ser facilmente confundida com outras lesões de tecido mole. Como diagnóstico diferencial destes tumores temos 0 fibroma, cisto dermóide, tumores menores de glândula salivar, mucocele, hemangioma, linfangioma, rabdomioma ou neuroma ${ }^{4,6,7,9,10}$. Nos casos em que o tumor apresenta coloração amarelada, a hipótese de lipoma prevalece, porém, quando a lesão tem coloração rósea como a mucosa adjacente, a hipótese de fibroma é mais plausível. Em nosso caso, a lesão apresentou-se de coloração rósea, semelhante a mucosa adjacente. A hipótese de fibroma foi considerada, porém, ao observar a flutuação do espécime após biopsia excisional, a sugestão de que a lesão se tratava de um lipoma foi considerada. No entanto, o diagnóstico definitivo foi obtido após análise microscópica da lesão.

Microscopicamente, a lesão é classificada de acordo com a matriz e células tumorais, podendo ser: lipoma simples, fibrolipoma, lipoma fusiforme, lipoma intramuscular, angiolipoma, condrolipoma, lipoma pleomórfico, lipoma mixóide e sialolipoma ${ }^{5,6}$. As características histopatológicas do lipoma clássico é uma lesão formada por tecido adiposo bem diferenciado, envolvido por uma capsula de tecido conjuntivo, que pode estar presente ou ausente. Quando o tecido conjuntivo fibroso passa a fazer parte da lesão, tem-se o fibrolipoma ${ }^{10}$. Um estudo retrospectivo realizado por Naruse et al. ${ }^{2}$ observou que o tipo histológico mais frequente foi o de lipoma simples, seguido por fibrolipoma, semelhante ao estudo realizado por Said-Al-Naief et $\mathrm{al}^{5}$, em que o tipo histológico mais comum foi o lipoma simples. Essa tendência é coincidente com o caso aqui apresentado, em que o diagnostico histopatológico tratou-se de lipoma simples.

O tratamento do lipoma intraoral, incluindo todas as variantes histológicas, é a excisão cirúrgica conservadora, com taxas de recidivas extremamente baixas $^{3,6,8}$. O lipoma intramuscular apresenta maiores taxas de recidivas devido seu crescimento infiltrativo. No entanto, essa variante é considerada rara na região bucomaxilofacial $^{7}$. Neste relato de caso, a biopsia excisional promoveu a remoção total da lesão, coincidindo com o tratamento proposto na literatura. Embora a lesão tenha crescimento lento, caso seja negligenciada pode chegar a tamanhos grotescos, podendo interferir na fala e na mastigação ${ }^{4,7}$. Nos exames periódicos de acompanhamento não foi observado recidiva da lesão.

\section{CONCLUSÃO}

Os lipomas intraorais são lesões raras e crescem lentamente. $\mathrm{O}$ cirurgião-dentista deve ter conhecimento das características clinicas dessa lesão, diferenciando-as de outras patologias de tecido mole, para que se possa estabelecer um plano de tratamento adequado.

\section{REFERÊNCIAS}

1. Juliasse LE, Nonaka CF, Pinto LP, Freitas Rde A, Miguel MC. Lipomas of the oral cavity: clinical and histopathologic study of 41 cases in a Brazilian population. Eur Arch Otorhinolaryngol. 2010; 267(3):459-65.

2. Naruse T, Yanamoto S, Yamada S, Rokutanda S, Kawakita A, Takahashi $\mathrm{H}$ et al. Lipomas of the oral cavity: clinicopathological and immunohistochemical study of 24 cases and 
review of the literature. Indian J Otolaryngol Head Neck Surg. 2015;67(Suppl 1):67-73.

3. Manor E, Sion-Vardy N, Joshua BZ, Bodner L. Oral lipoma: analysis of 58 new cases and review of the literature. Ann Diagn Pathol. 2011;15(4):257-61.

4. Avelar LR, Carvalho RWF, Falcão PGCB, Antunes AA, Andrade ESS et al. Lipomas da Região Oral e Maxilofacial: Estudo Retrospectivo de 16 Anos no Brasil. Rev Port Estomatol Cir Maxilofac 2008;49(4):207-11.

5. Said-Al-Naief N, Zahurullah FR, Sciubba JJ. Oral spindle cell lipoma. Ann Diagn Pathol. 2001;5(4):207-15.

6. Egido-Moreno S, Lozano-Porras AB, Mishra S, Allegue-Allegue M, Marí-Roig A, López-López J. Intraoral lipomas: Review of literature and report of two clinical cases. J Clin Exp Dent. 2016;8(5):e597-603.

7. A RK, N PN, Y S, A VK, D KK. Intraoral lipoma: a rare case report and review of literature. J Clin Diagn Res. 2013;7(12):3090-91.

8. Raj M, Ramadoss T, Anuradha G, Devi S. Intraoral lipoma: review of literature and case report. J Indian Acad Oral Med Radiol. 2012; 24(1): 36-38

9. Carvalho MF, Junqueira TP, Souza RR, Capistrano HM, Chaves MGAM.The importance of early diagnosis of large lipomas in the maxillofacial region. Rev Cubana Estomatol. 2011;48(1):77-83

10. Noro Filho GA, Caputo BV, Santos CC, Souza RS, Giovani EM, Scabar LF et al. Diagnosis and treatment of intraoral lipoma: a case report. J Health Sci Inst. 2010;28(2):129-31

\section{CONFLITO DE INTERESSES}

Os autores declaram não haver conflitos de interesse.

\section{AUTOR PARA CORRESPONDÊNCIA}

Naíza Menezes Medeiros Abrahim

Rua 30, Quadra 35, n-4, Condominio Vila Verde II.

Manaus/AM. Telefone: (55) 92 99962.0222.

E-mail: naizamedeiros@hotmail.com

Submetido em 08/08/2019

Aceito em 26/03/2020 